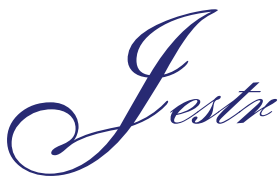

Journal of Engineering Science and Technology Review 2 (1) (2009)151-156

JOURNAL OF

Engineering Science and

Technology Review

Research Article

www.jestr.org

\title{
Morphological feature selection and neural classification for electronic components
}

\author{
Dionysios Lefkaditis and Georgios Tsirigotis* \\ Kavala Institute of Technology, 65404, St.Lucas, Kavala, Greece.
}

Received 21 September 2009; Revised 4 November 2009; Accepted 18 November 2009

\begin{abstract}
This paper presents the development procedure of the feature extraction and classification module of an intelligent sorting system for electronic components. This system was designed as a prototype to recognise six types of electronic components for the needs of the educational electronics laboratories of the Kavala Institute of Technology. A list of features that describe the morphology of the outline of the components was extracted from the images. Two feature selection strategies were examined for the production of a powerful yet concise feature vector. These were correlation analysis and an implementation of support vector machines. Moreover, two types of neural classifiers were considered. The multilayer perceptron trained with the back-propagation algorithm and the radial basis function network trained with the K-means method. The best results were obtained with the combination of SVMs with MLPs, which successfully recognised $92.3 \%$ of the cases.
\end{abstract}

Keywords: Recognition, Neural Network, Classification.

\section{Introduction}

This paper gives an account of the construction of an intelligent sorting system for electronic components. Specific focus is given on the comparison of two feature selection methods used to optimise the morphological feature vector. Correlation analysis and support vector machines were considered as they represent two very different approaches for feature selection. The performance of these methods was measured through the successful recognition rates of two neural classifiers, the multilayer perceptron and the radial basis function network. The best performing combination of methods was then proposed as the classification module of the sorting system.

Correlation analysis was utilised to discover any underlying relationships between the features, in essence to find out if they refer to the same property of the sample's outline. Therefore, unwanted repetitions of information were discarded, thus the feature vector was shrunk and simplified with minimum loss of its descriptive ability.

Support vector machines (SVMs) were examined as an alternative technique for feature selection. Here, SVMs were set to perform a supervised classification task on the data. The focus was not to optimise the classification performance but to quantify the discrimination ability of the features and sort them on this basis. The sorting criterion was the squared weight of each variable defined by the support vectors.

The multilayer perceptron class of neural networks consists of

* E-mail address: tsirigo@teikav.edu.gr

ISSN: 1791-2377 (C) 2009 Kavala Institute of Technology. All rights reserved. multiple layers of computational units, interconnected in a feedforward way. Each neuron in one layer has directed connections to the neurons of the subsequent layer and applies a sigmoid function as an activation function [9]. These networks were trained using the supervised back-propagation algorithm [3].

A radial basis function ( $\mathrm{RBF}$ ) is a function, whose value is governed by the distance from a centre. RBFs have been applied in the area of neural networks, where they may be used as a replacement for the sigmoidal hidden layer transfer characteristic in multilayer perceptrons. The RBF chosen is usually a Gaussian distribution function [3]. The training of these networks was to calculate the coordinates of the Gaussian centres by the unsupervised K-means clustering method [5].

\section{Materials and methods \\ 2.1 Image acquisition and processing}

The acquisition of images took place at the laboratories of the Fisheries Research Institute at Nea Peramos, Kavala, Greece. The institute kindly provided all the necessary equipment and laboratory space for the needs of this study.

The device characteristics that were desired for this application were the adequate image resolution of the sensor, good quality optics, the space available between the objective lens and the sample, the adaptability to various lighting techniques and the speed 
of operation. After the examination of several available devices the one that offered the most attractive combination of the above was a high precision desktop photographic system with a standalone control unit made by Nikon.

The use of back illumination was found to efficiently deal with problems such as the presence of shadows and the reflections of metallic surfaces. The sample was situated in between the light source and the image sensor. In this manner, the sample appeared to be dark in a bright background. Another advantage of this technique was that the foreground-to-background contrast was very high. The disadvantage of decreased colour information was not important for the specific application.

A Leica stereoscopic microscope stage in conjunction with a Leica L2 fibre optic light source and a transparent sample holder was used to achieve the back-illumination lighting. A piece of A4 paper was placed between the sample and the sample holder to diffuse the light and create an evenly illuminated background. This technique gave excellent results and minimised the need for advanced image-processing algorithms to extract the sample outlines. The image acquisition setup can be seen in figure 1 .

In this study, custom code was developed to automatically perform the image processing and feature extraction. The open-source and Java-based application ImageJ was used as a platform for this task [1]. The images were scaled and cropped, and the colour information was discarded by converting the images from 24 bit colour to 8 bit greyscale to reduce the unnecessary volume of data and speed up the processing. Image segmentation was performed using the "Minimum Auto Threshold" algorithm [11]. The mathematical morphology algorithm "closing" was applied to fix any broken lines as a result of local miscalculation of the threshold limit [13]. This ensured the reliable production of image measurements for the extraction of the features.

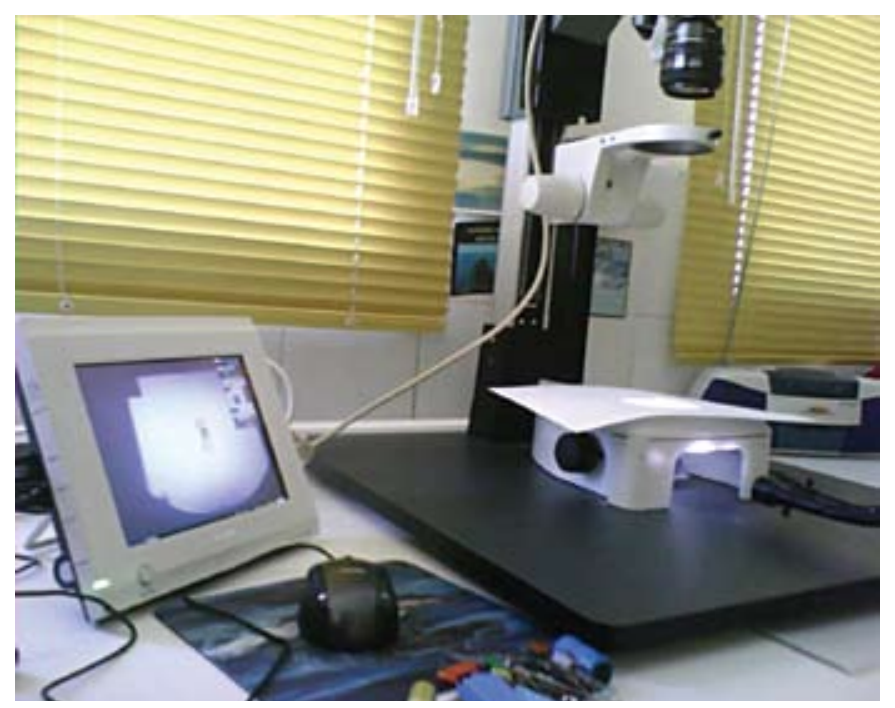

Figure 1. Image acquisition subsystem

\subsection{Extraction of morphological features}

Feature extraction is performed as a way to reduce the dimensionality of the data produced by the image sensor. In the computer vision field, each pixel represents one data dimension. The images used in this study provided data of 200340 dimensions as the images used were $477 \times 420$ pixels. It is impractical to feed data of such high dimensionality into a classifier. A long list of candidate features was calculated in order to form a powerful input vector for the neural classification module of the system. The features attempted to describe the morphology of the outline shape of the electronic components. Once extracted and optimised, the vector would be used to train and validate the classifier. The list of candidate features contained:

\section{- Area.}

This is calculated as the sum of pixels that are enclosed within the boundaries of the sample. It is then converted in calibrated units, such as $\mu \mathrm{m}^{2}$ to enable direct comparison among sample images that are not acquired using the same lens set-up on the stereoscope. It may be useful to give a measure of absolute size.

\section{- Perimeter.}

This is calculated as the sum of the pixels that form the boundary of the sample. It is also converted in calibrated units $(\mu \mathrm{m})$ according to the lens set-up of the stereoscope. It offers a measure of size as well as a measure of outline roughness.

- Maximum Feret's diameter.

This is defined as the greatest distance possible between any two points along the boundary of the sample or the greatest distance between two vertical lines tangential to the ends of the particle [1]. It is useful in conjunction with magnification data from the stereoscope to give a measure of actual size scale of the sample.

\section{- Circularity.}

A simple shape factor based on the projected area of the sample and the overall perimeter of the projection according to:

$$
C=\frac{4 \pi A}{P^{2}}
$$

where $\mathrm{A}$ is the area and $\mathrm{P}$ is the perimeter of the sample.

Values range from 1 , for a perfect circle, to 0 for a line. It is useful to give an impression of elongation as well as roughness of the sample's shape. Other significant characteristics of this feature are that it is invariant to scale, translation and rotation [4].

\section{- Solidity.}

It is the ratio of the area of the sample (A) over the area of its convex hull $\left(\mathrm{A}_{\text {ConvexHull }}\right)[6]$. The formula for solidity is:

$$
\text { Solidity }=\frac{A}{A_{\text {ConvexHull }}}
$$

- $\mathrm{R}$ factor.

The $\mathrm{R}$ factor measures the perimeter of the convex hull of the sample over its maximum Feret's diameter [6]. $\mathrm{R}$ factor is given by:

$$
R \text { factor }=\frac{P_{\text {ConvexHull }}}{(\pi \text { Feret })}
$$

\section{- MBR aspect ratio.}

This is the ratio of the sides of the "minimum bounding rectangle" (MBR) that includes the sample. The minimum bounding rectangle, also known as "bounding box", is an expression of the 
maximum extents of a 2-dimensional object (e.g. point, line, polygon) within its 2-D (x,y) coordinate system, in other words $\min (\mathrm{x})$, $\max (\mathrm{x}), \min (\mathrm{y}), \max (\mathrm{y})[7]$. It may be useful to give a measure of the elongation of the samples. The formula for aspect ratio is:

$$
\text { Aspect ratio }=\frac{\mathrm{x}_{\max }-\mathrm{x}_{\text {min }}}{\mathrm{y}_{\text {max }}-\mathrm{y}_{\text {min }}}
$$

\section{- Convexity.}

It is the ratio of the perimeter of the convex hull of the sample $\left(P_{\text {convexHull }}\right)$ over the actual perimeter of the sample $(P)$. For planar objects, the convex hull may be easily visualized by imagining an elastic band stretched open to encompass the given object; when released, it will assume the shape of the required convex hull [8]. The formula for convexity is:

$$
\text { Convexity }=\frac{P_{\text {ConvexHull }}}{P}
$$

- Concavity.

This is the subtraction of the area of the sample $(A)$ from the area covered by its convex hull $\left(A_{\text {ConvexHull }}\right)[6]$. The formula used for concavity is:

$$
\text { Concavity }=A_{\text {ConvexHull }}-A
$$

- Rectangularity.

This is the ratio of the area of the sample over the area of its MBR [10]. It approaches 0 for cross-like objects, 0.5 for squares, $\pi / 4$ for circles and 1 for long rectangles [6]. The formula used for rectangularity is:

$$
\text { Rectangularity }=\frac{A}{A_{M B R}}
$$

\section{- Feret's aspect ratio}

This is the ratio of the maximum Feret's diameter over the maximum diameter of the object which is perpendicular to the one of Feret's, in other words the breadth of the object. It is given by:

$$
A R=\frac{\text { Feret }}{\text { breadth }}
$$

\section{- Roundness.}

It is given by the area of the sample over the square of its maximum Feret's diameter [6]. The formula for roundness is:

$$
\text { Roundness }=4 \frac{A}{\pi F \text { eret }^{2}}
$$

- Compactness

This feature also associates the area of the sample over its maximum Feret's diameter. The formula to calculate it is:

$$
\text { Compactness }=\frac{\sqrt{\frac{4}{\pi} A}}{\text { Feret }}
$$

Area, perimeter and maximum Feret's diameter were extracted to enable the calculation of the rest of the features and were not included in the feature vector.

\subsection{Feature selection}

The extraction of the 13 features mentioned above produced a multidimensional set of data. The exploration of the data set can give a valuable insight of the expected behaviour of the end system and therefore assist further improvement of its performance [10]. This knowledge can be gained by finding out the internal relationships between the features and the quantification of their descriptive power.

The feature selection and the classification tasks of this study were performed with the widely respected open-source software package WEKA [14].

In multidimensional data sets, correlation analysis is computed by calculating the Pearson coefficient between every combination of features. The resulting values were then arranged in a table format called correlation matrix. In the event that the value of the coefficient was higher than \pm 0.85 , the correlation was considered too high and one of the two features was excluded from the feature vector.

The use of the correlation matrix simplified the detection of high correlating features and the decision of which one should be excluded. Table 1 illustrates the correlation matrix:

Table 1. Correlation matrix of the complete feature vector

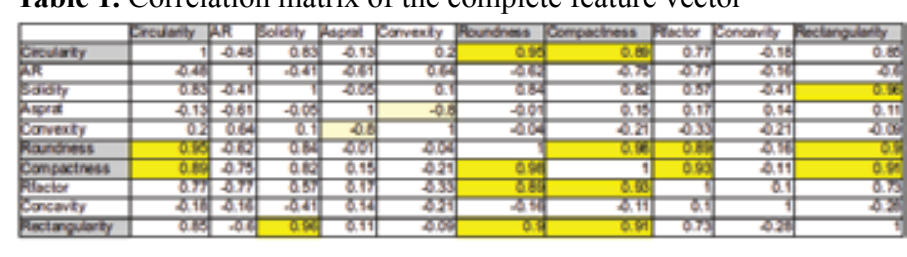

In the correlation matrix, the values that lie above the limit are highlighted in yellow and the features considered for exclusion in grey. After the selection process six features remained in the optimised feature vector. These are shown in table 2:

Table 2. List of selected features by correlation analysis

\begin{tabular}{c}
\hline Feature name \\
\hline Feret's aspect ratio \\
Solidity \\
MBR aspect ratio \\
Convexity \\
R factor \\
Concavity \\
\hline
\end{tabular}

A good example for illustrating the relationship between two high correlating features was "Roundness" and "Compactness". A plot of their values against each other is presented in figure 2 , which it is very close to a straight line as they are highly correlated: 


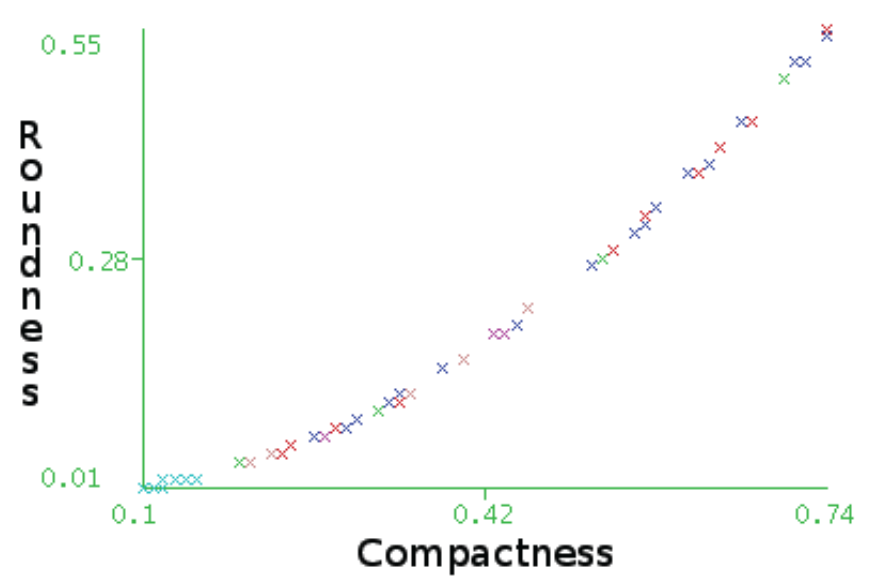

Figure 2. Plot of Roundness against Compactness showing their high correlation

As mentioned before, SVMs were used as supervised alternative feature selection technique to the unsupervised correlation analysis. After the classification, features were sorted based on their discriminating ability. Table 3 presents the results of this technique:

Table 3. Features sorted by their discriminating ability using SVMs

\begin{tabular}{cc}
\hline Discriminating ability & Feature name \\
\hline 1 & Solidity \\
2 & R factor \\
3 & Feret's aspect ratio \\
4 & Convexity \\
5 & Rectangularity \\
6 & MBR aspect ratio \\
7 & Compactness \\
8 & Circularity \\
9 & Concavity \\
10 & Roundness \\
\hline
\end{tabular}

For direct comparison with correlation analysis the six most powerful features were selected. It is worth noting that the two optimised feature vectors are very similar to each other. 5 out of the 6 features selected were the same. SVMs selected rectangularity instead of concavity that was selected by correlation analysis.

The actual performance of the optimised vectors was measured through the performance of classification module of the system.

\subsection{Classifier training and validation}

In this study, two neural classifiers were considered that use very different training approaches. The multilayer perceptrons (MLPs) are trained with the supervised back-propagation algorithm. The radial basis function networks (RBFNs) use the K-means clustering method to define the centres of their Gaussian functions. The $\mathrm{K}$-means is a well-known unsupervised technique.

Initially, these networks were trained with the full 10-element feature vector so as to set it as the benchmark to which the optimised feature vector would be compared. Therefore, the classifier validation served two purposes, the identification of the most powerful feature selection technique and the determination of the most suitable classifier for this application.

Generally in the classification neural networks, it is common practice to use the number of elements of the feature vector as the number of neurons of the input layer and the number of neurons of the output layer to be the number of classes of the data. In this application for the MLP, the number of neurons was found in a trial and error manner. However, the approach that gave the best results was to use the half of the sum of the rest of the nodes of the network as the number of hidden nodes. For example, in the case of the full feature vector, there were 10 input and 6 output nodes that made 8 nodes in the hidden layer. Moreover, the best learning rate and the momentum factor of the back-propagation training were found to be 0.8 and 0.3 respectively. The networks were trained for 1000 cycles as longer training did not provide lower error rates. The data, consisting of 87 sets in total, was split in $70 \%$ for training and $30 \%$ for validation.

After the training sessions, the classifier evaluation yielded an overall performance of $92.3 \%$. The various classes gave differing degrees of successful recognitions that can be described by two metrics. True Positive (TP) rate is the actual rate of successful recognitions and False Positive rate (FP) shows the percentage of mistakenly classified cases per class. Table 4 presents the detailed results.

The class Elcap refers to electrolytic capacitors, Cercap to square and round ceramic capacitors, Res to resistors, Trans to transistors and PowTrans to power transistors.

Table 4. Detailed MLP classification performance per data class

\begin{tabular}{ccc}
\hline Class & TP & FP \\
\hline Elcap & 0,857 & 0,053 \\
Cercap sq & 0,833 & 0 \\
Cercap rd & 1 & 0 \\
Res & 1 & 0 \\
Trans & 1 & 0 \\
PowTrans & 1 & 0,045 \\
\hline
\end{tabular}

An alternative presentation method of these results is the contingency table, where it the misclassification are shown more clearly. The diagonal of the table depicts the true successful recognitions. The contingency table is table 5 .

Table 5. Contingency table of the MLP classifier

\begin{tabular}{|c|c|c|c|c|c|c|}
\hline \multicolumn{7}{|c|}{ Classes classified as... } \\
\hline Elcap & $\begin{array}{c}\text { Cer- } \\
\text { cap sq }\end{array}$ & $\begin{array}{l}\text { Cer- } \\
\text { cap rd }\end{array}$ & Res & Trans & $\begin{array}{c}\text { Pow } \\
\text { Trans }\end{array}$ & Classes \\
\hline 6 & 0 & 0 & 0 & 0 & 1 & Elcap \\
\hline 0 & 5 & 1 & 0 & 0 & 0 & Cercap sq \\
\hline 0 & 0 & 1 & 0 & 0 & 0 & Cercap rd \\
\hline 0 & 0 & 0 & 5 & 0 & 0 & Res \\
\hline 0 & 0 & 0 & 0 & 3 & 0 & Trans \\
\hline 0 & 0 & 0 & 0 & 0 & 4 & PowTrans \\
\hline
\end{tabular}

The table below suggests that the overall classification rate was very high. However, the electrolytic capacitors can be confused with power transistors and the square ceramic capacitors as round 
ones. Considering the nature of feature within the input vector, it can be realised that these classes have indeed very similar shape and outline properties.

For the RBFNs, the number of Gaussian functions needs to be far greater than the expected data clusters in the feature space, so as for the network to able to map the data with enough resolution. Here they were trained with 30 nodes in their hidden layer as higher number of Gaussians did not offer better results.

As it was expected the unsupervised RBFNs did not match the performance of the MLPs. They gave an overall successful recognitions rate of $65.4 \%$. Therefore, they are not recommended for this application.

The performance of the MLP networks trained with the optimised feature vectors was very high. For both the optimisation methods it matched the overall successful recognitions rate of 92.3\% and the per class rates. This shows that both of the methods achieved to discard only repetitive and unneeded information. Therefore, there can be more than one optimised feature vectors for a specific application.

The RBFNs also gave interesting results when trained with the optimised vectors. The correlation analysis vector offered an increase of $3.84 \%$ to the overall successful recognitions rate, making it $69.2 \%$. This was probably because the unsupervised networks are very prone to confusing and repetitive data.

Training the RBFNs with a supervised feature selection technique dramatically improved their performance. They achieved to give $84.6 \%$ successful recognitions rate, an increase of $19.2 \%$. Therefore, the SVMs offer a far more powerful feature vector. This method is based on actual supervised classification, which sorts the features according to their real descriptive abilities and it is not dependant on the discovery of underlying subjective relationships between the features. It is therefore, a matter of comparison between a supervised and an unsupervised methodology on the same data set, where it is expected that the supervised one will be superior.

In order to examine whether a six element feature vector is the optimum size for this application, one more element was excluded from both the optimised vectors. The performance decreased for the MLPs and the RBFNs for both optimisation methods. It needs to be noted that SVMs gave a smaller performance decrease for both network types. This means that it is also more reliable than correlation analysis on the specific application. Therefore, here it is the preferred method.

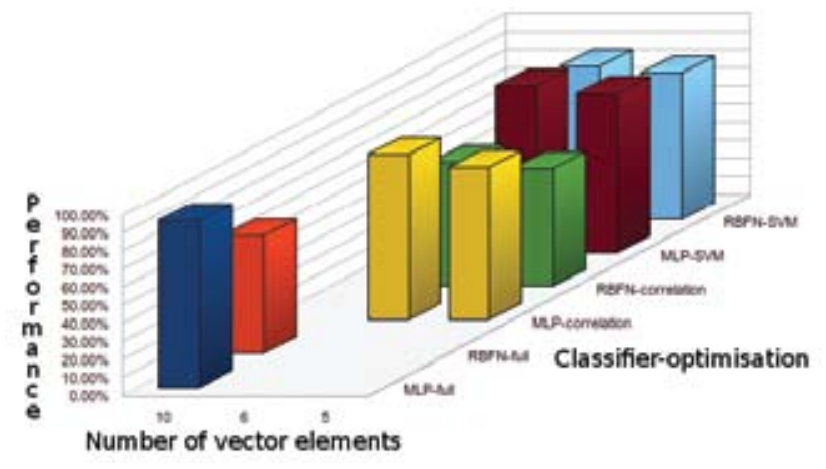

Figure 3. Concentrative graph of classifier performances
All the above results are presented graphically in figure 3 and documented in detail in table 6 .

\section{Conclusions}

This paper presented a thorough account of the feature selection process and the development of the neural classifier a computer vision system for the recognition of electronic components.

Table 6. Concentrative table of classification results

\begin{tabular}{ccccccc}
\hline \multicolumn{3}{c}{ Optimisation } & & & & \\
$\begin{array}{c}\text { Vector } \\
\text { element } \\
\text { number }\end{array}$ & none & & $\begin{array}{c}\text { Cor- } \\
\text { relation } \\
\text { analysis }\end{array}$ & & SVM & \\
\hline 10 & $92.31 \%$ & $65.38 \%$ & - & - & - & - \\
6 & - & - & $92.31 \%$ & $69.23 \%$ & $92.31 \%$ & $84.62 \%$ \\
5 & - & - & $84.62 \%$ & $65.38 \%$ & $88.46 \%$ & $80.77 \%$ \\
Classifier & MLP & RBFN & MLP & RBFN & MLP & RBFN \\
\hline
\end{tabular}

The system was designed with the generic model of the modular machine vision system by Awcock and Thomas [2] as a guide. The image acquisition equipment was a Nikon high precision desktop photographic system with a standalone control unit as it offered high quality images, adaptability to various lighting techniques and speed of operation. The lighting technique used was back-illumination so that the creation of shadows and reflections of metallic surfaces can be avoided.

The processing of the images and the feature extraction required were implemented as a custom ImageJ macro code. A list of 10 morphological features based on the outline of the samples was calculated to reduce the dimensionality of the image data.

Two types of neural classifiers were considered, the multilayer perceptrons (MLPs) and the radial basis function networks (RBFNs). These classifiers were initially trained with the full feature vector to act as a benchmark. Two feature vector optimisation methods were examined, correlation analysis and SVMs. Generally, the MLP classifiers performed much better than the unsupervised RBFNs. For the MLPs, both feature selection methods matched the performance given by the full feature vector. However, for the RBFNs, the SVMs presented a far better performance than with both the full and the correlation analysis optimised feature vector. They are better suited to this application because it is a supervised feature selection technique on a supervised classification problem. Therefore, the SVMs were considered as a more reliable feature selection method for the final system even though it offers the same performance as with the other feature vectors for the chosen MLP classifier.

Training with a further reduced five-element feature vector showed a decrease in performance for both MLPs and RBFNs. This proved that the vector size of six elements was indeed the right vector size for this application.

As a final conclusion, for the above reasons the combination of the SVM optimised six-element feature vector with the MLP neural networks was considered to give the most powerful classification module for this computer vision system. 


\section{References}

1. Abramoff, M. D., Magelhaes, P. J., Ram, S. J., 2004. "Image Processing with ImageJ”. Biophotonics International, volume 11, issue 7, pp. 36-42.

2. Awcock, G. J. \& Thomas, R., 1995. Applied image processing, Hightstown, NJ, USA: McGraw-Hill, Inc.

3. Bishop, C. M., 1995. Neural Networks for Pattern Recognition, Oxford, UK: Oxford University Press.

4. Bouwman, A. M., Bosma, J. C., Vonk, P., Wesselingh, J. A. \& Frijlink, H.W. (2004) Which shape factor (s) best describe granules. Powder Technology, 146 (1-2), pp.66-72.

5. Duda, R, Hart, P \& Stork, D., 2001, Pattern classification, Wiley New York.

6. Eid, R. \& Landini, G., 2006. Oral Epithelial Dysplasia: Can quantifiable morphological features help in the grading dilemma? In Luxembourg.

7. Glasbey, C. \& Horgan, G., 1995. Image analysis for the biological sciences, New York: Wiley.

8. Graham, R., 1972. An Efficient Algorithm for Determining the Convex
Hull of a Finite Planar Set. Information Processing Letters, 1(4), 132-133.

9. Haykin, S., 1998. Neural Networks: A Comprehensive Foundation, Prentice Hall PTR Upper Saddle River, NJ, USA.

10. Lefkaditis, D., Awcock, G. J. \& Howlett, R. J., 2006. Intelligent Optical Otolith Classification for Species Recognition of Bony Fish. In International Conference on Knowledge-Based \& Intelligent Information \& Engineering Systems. Bournemouth, UK: Springer-Verlag, pp. 1226-1233.

11. Prewitt, J. \& Mendelsohn, M., 1966. The analysis of cell images. Ann. NY Acad. Sci, 128, 1035-1053.

12. Rosin, P. L., 2003. Measuring shape: ellipticity, rectangularity, and triangularity. Machine Vision and Applications, 14(3), 172-184.

13. Serra, J., 1983. Image analysis and mathematical morphology. Academic Press, Inc. Orlando, FL, USA

14. Witten, I and Frank, E, 2005. "Data Mining: Practical machine learning tools and techniques", 2nd Edition, Morgan Kaufmann, San Francisco. 